\title{
Enumeration Schemes for Permutations Avoiding Barred Patterns
}

\author{
Lara Pudwell* \\ Department of Mathematics and Computer Science \\ Valparaiso University, Valparaiso, IN 46383 \\ Lara.Pudwell@valpo.edu
}

Submitted: May 18, 2008; Accepted: Feb 10, 2010; Published: Feb 15, 2010

Mathematics Subject Classification: 05A05

\begin{abstract}
We give the first comprehensive collection of enumeration results for permutations that avoid barred patterns of length $\leqslant 4$. We then use the method of prefix enumeration schemes to find recurrences counting permutations that avoid a barred pattern of length $>4$ or a set of barred patterns.
\end{abstract}

\section{Introduction}

Let $q=q_{1} \cdots q_{m}$ be a finite string of numbers. The reduction of $q$, denoted $\operatorname{red}(q)$, is the string obtained by replacing the $i$ th smallest letter(s) of $q$ with $i$. For example, the $\operatorname{red}(2674425)=1452213$. Given two permutations $p \in S_{n}, q \in S_{m}$, we say $p$ contains $q$ as a pattern if there exist $1 \leqslant i_{1}<\cdots<i_{m} \leqslant n$ such that $\operatorname{red}\left(p_{i_{1}} \cdots p_{i_{m}}\right)=q$. Otherwise $p$ avoids $q$. This definition of pattern avoidance appears in the characterization of 1stack sortable permutations [11] and the characterization of smooth Schubert varieties 6]. Further, it introduces an interesting and well-studied enumeration problem; namely, count the elements of the set $S_{n}(Q)=\left\{p \in S_{n} \mid p\right.$ avoids $q$ for all $\left.q \in Q\right\}$.

The focus of this paper is not the study $S_{n}(Q)$, but a variation of pattern avoidance given by the following definitions. Given $q^{\prime} \in S_{m}, b \in\{0,1\}^{m}$, the barred permutation $q$ is the permutation obtained by copying the entries of $q^{\prime}$ and putting a bar over $q_{i}^{\prime}$ if and only if $b_{i}=1$. Write $\overline{S_{m}}$ for the set of all barred permutations of length $m$. For example, the complete set of barred permutations of length 2 is $\{12, \overline{1} 2,1 \overline{2}, \overline{12}, 21, \overline{2} 1,2 \overline{1}, \overline{21}\}$.

${ }^{*}$ The author thanks an anonymous referee for several useful suggestions that simplified the organization of this paper. 
A barred permutation compactly encodes two permutations, one of which contains the other. In particular, let $\underline{q}$ be the permutation formed by deleting all barred letters of $q$ and then reducing the remaining (unbarred) letters, and let $\bar{q}$ be the permutation formed by all letters of $q$, with or without bars. For $p \in S_{n}, q \in \overline{S_{m}}$, we say $p$ contains $q$ as a barred pattern if every instance of $q$ in $p$ is part of an instance of $\bar{q}$ in $p$. In this case, we may say every instance of $q$ extends to an instance of $\bar{q}$. For example if $q=\overline{1} 32$, we have $\underline{q}=\operatorname{red}(32)=21$ and $\bar{q}=\overline{1} 32 . p$ avoids $q$ if and only if every decreasing pair of numbers in $p$ has a smaller number preceding it.

This variation of pattern avoidance also appears in several interesting applications.

- A permutation is two stack sortable if and only if it avoids 2341 and $3 \overline{5} 241$ [1].

- A permutation is forest-like if and only if it avoids the patterns 1324 and $21 \overline{3} 54$ [2]. These permutations also characterize locally factorial Schubert varieties [12].

Beyond the special cases of barred pattern avoidance relevant to these applications, little is known beyond the work of Callan, where he completely enumerates permutations avoiding a single pattern of length 4 with one bar [3], and deals with the special case of $\{3 \overline{5} 241\}$-avoiding permutations [4]. The goal of this paper is to consider the problem of barred pattern avoidance in a more general and comprehensive context. We consider barred permutations of any length and with any number of bars. Several preliminary results are given, and we completely characterize permutations avoiding a barred pattern of length $\leqslant 5$, before we modify the method of prefix enumeration schemes to the case of barred pattern avoidance, and discuss its success rate.

\section{Enumeration}

Before we consider results for specific sets of barred patterns, we derive a series of useful lemmas

Lemma 1. Let $q \in \overline{S_{m}}$, such that every letter of $q$ is barred. Then $S_{n}(\{q\})$ is the set of permutations that contain $\bar{q}$.

Proof. Notice that $\underline{q}=\emptyset$. That is for $p$ to avoid $q$ every instance of the empty permutation must be a part of a copy of $\bar{q}$, i.e. $p$ contains $\bar{q}$.

More specifically, this lemma illustrates that, in some sense, barred pattern avoidance bridges the gap from standard pattern avoidance (no bars) to standard pattern containment (all possible bars), with a range of intermediate cases. However, as the following propositions illustrate, a number of these intermediate cases may also be equivalent to standard pattern avoidance.

Lemma 2. Let $q \in \overline{S_{m}}$ such that $q_{i}$ is barred and either (i) $q_{i+1}$ is unbarred with $q_{i+1}=$ $q_{i} \pm 1$, or (ii) $q_{i-1}$ is unbarred with $q_{i-1}=q_{i} \pm 1$. Then,

$$
S_{n}(\{q\})=S_{n}(\{\underline{q}\}) .
$$


Proof. Clearly, if $p$ avoids $\underline{q}$, then it avoids $q$ since there are no instances of $\underline{q}$ to expand to an instance of $\bar{q}$. Thus,

$$
S_{n}(\{\underline{q}\}) \subseteq S_{n}(\{q\}) .
$$

On the other hand, without loss of generality assume that $q_{i+1}$ is unbarred, $q_{i}=q_{i+1} \pm 1$, $p$ avoids $q$ and there is an instance of $q$ in $p$ that extends to an instance of $\bar{q}$. Choose the extension to $\bar{q}$ that uses the leftmost possible element $p^{*}$ of $p$ for $q_{i}$. Now, this instance of $\bar{q}$ necessarily contains an at least two instances of $q$ : the original instance that was extended to $\bar{q}$, and an instance of $q$ formed by taking the first instance, deleting the letter playing the role of $q_{i+1}$ and replacing it with $p^{*}$. This second instance of $\underline{q}$ cannot be extended to $\bar{q}$. So every element of $S_{n}(\{q\})$ already avoids $\underline{q}$. That is,

$$
S_{n}(\{q\}) \subseteq S_{n}(\{\underline{q}\})
$$

The next lemma provides a specific enumeration result that will prove useful in the following sections of this paper.

Lemma 3. Let $i d_{k}=12 \cdots k$, and let $p+k$ denote the string where $k$ is added to each entry of permutation $p$. Then

$$
\left|S_{n}\left(\left\{\overline{i d_{k}}(21+k) \overline{i d_{l}+k+2}\right\}\right)\right|=(n-k-l) ! \text { for } n \geqslant 0 .
$$

Proof. In particular we will show that $S_{n}\left(\left\{\overline{i d_{k}}(21+k) \overline{i d_{l}+k+2}\right\}\right)$ is exactly the set of permutations that begin with $12 \cdots k$ and end with $(n-l+1) \cdots n$.

Clearly, if $p$ begins with $12 \cdots k$ and ends with $(n-l+1) \cdots n$, then every 21 pattern extends to a $i d_{k}(21+k)\left(i d_{l}+k+2\right)$ pattern, as desired.

Now, if $p$ does not begin with $12 \cdots k$, then either (i) $p$ begins with an increasing run of $k$ letters that does not include some number in the set $\{1, \ldots, k\}$ (and thus $p_{k}$ is part of a 21 pattern), or (ii) the first $k$ letters of $p$ contain a 21 pattern. In either case, $p$ contains an instance of 21 that cannot be extended to $i d_{k}(21+k)\left(i d_{l}+k+2\right)$. A similar argument holds if $p$ does not end with $(n-l+1) \cdots n$.

Now that we know $S_{n}\left(\left\{\overline{i d_{k}}(21+k) \overline{i d_{l}+k+2}\right\}\right)$ is exactly the set of permutations that begin with $12 \cdots k$ and end with $(n-l+1) \cdots n$, we may place any permutation of $\{k+1, \ldots, n-l\}$ in positions $p_{k+1} \cdots p_{n-l}$ and obtain an element of $S_{n}\left(\left\{\overline{i d_{k}}(21+\right.\right.$ $\left.\left.k) \overline{i d_{l}+k+2}\right\}\right)$, so indeed $\left|S_{n}\left(\left\{\overline{i d_{k}}(21+k) \overline{i d_{l}+k+2}\right\}\right)\right|=(n-l-k)$ !.

Finally, we eliminate the case of having bars on all but one letter by the following observation.

Lemma 4. Suppose that $q \in \overline{S_{m}}$ with only one unbarred letter. Then $\left|S_{n}(\{q\})\right|=0$ for all $n \geqslant 1$

Proof. Notice that avoiding $q$ means that every instance of a 1 pattern expands to an instance of $\bar{q}$. Without loss of generality, assume that $q$ has barred entries after the lone unbarred letter. Then the final entry of any permutation is a copy of 1 that does not expand to $\bar{q}$. 
We now consider permutations avoiding patterns of length $1,2,3,4$, and 5 in turn, noting that many results follow almost directly from Lemmas 1, 2, and 3. With the exception of the work of Callan [3] for patterns of length 4 with 1 bar, this is the first comprehensive list of such results.

\subsection{Avoiding barred patterns of length 1 or 2}

We begin with avoiding patterns of length 1 .

It is well known that $\left|S_{n}(\{1\})\right|=\left\{\begin{array}{ll}1 & n=0 \\ 0 & n \geqslant 1\end{array}\right.$.

We now see from Lemma 1 that $\left|S_{n}(\{\overline{1}\})\right|=\left\{\begin{array}{ll}0 & n=0 \\ n ! & n \geqslant 1\end{array}\right.$.

For patterns of length 2, we observe that the Wilf equivalences

$$
\left|S_{n}(\{q\})\right|=\left|S_{n}\left(\left\{q^{r}\right\}\right)\right|=\left|S_{n}\left(\left\{q^{c}\right\}\right)\right|=\left|S_{n}\left(\left\{q^{-1}\right\}\right)\right|
$$

extend to barred patterns in the obvious way, where $q^{r}$ denotes $q$ reverse, $q^{c}$ denotes $q$ complement, and $q^{-1}$ denotes $q$ inverse [8].

Thus, we already have $\left|S_{n}(\{12\})\right|=\left|S_{n}(\{21\})\right|=1, n \geqslant 0$.

Further, by Lemma 1, we have $\left|S_{n}(\{\overline{12}\})\right|=\left|S_{n}(\{\overline{21}\})\right|=n !-1$.

Finally, $\left|S_{n}(\{\overline{1} 2\})\right|=\left|S_{n}(\{2 \overline{1}\})\right|=\left|S_{n}(\{1 \overline{2}\})\right|=\left|S_{n}(\{\overline{2} 1\})\right|=\left|S_{n}(\{1\})\right|$, where the first and third equalities are by reversal, the second equality is by complement, and the final equality is by Lemma 2 .

\subsection{Avoiding barred patterns of length 3}

It is well known that $\left|S_{n}(\{q\})\right|=\frac{\left(\begin{array}{c}2 n \\ n\end{array}\right)}{n+1}$ where $q$ is any unbarred pattern of length $3[8]$.

Thus, $\left|S_{n}(\{\bar{q}\})\right|=n !-\frac{\left(\begin{array}{c}2 n \\ n\end{array}\right)}{n+1}$ where $\bar{q}$ is any pattern of length 3 with all bars.

By Lemma 4 it only remains to consider the case of patterns with 1 bar. The trivial Wilf equivalences and Lemma 2 give:

$$
\begin{gathered}
\left|S_{n}(\{\overline{1} 23\})\right|=\left|S_{n}(\{32 \overline{1}\})\right|=\left|S_{n}(\{12 \overline{3}\})\right|=\left|S_{n}(\overline{3} 21)\right|=\left|S_{n}(21)\right|, \text { and } \\
\left|S_{n}(\{1 \overline{2} 3\})\right|=\left|S_{n}(3 \overline{2} 1)\right|=\left|S_{n}(21)\right| .
\end{gathered}
$$

It is enough to consider the pattern 132 with bars on various elements to complete the characterization. If there is a bar on 3 or 2, we may make use of Lemma 2, so the remaining interesting case is that of $S_{n}(\{\overline{1} 32\})$. However, we know that $\left|S_{n}(\{\overline{1} 32\})\right|=(n-1)$ ! for all $n \geqslant 0$ by Lemma 3 .

We have now finished the enumeration of permutations avoiding barred patterns of length $\leqslant 3$. 


\subsection{Avoiding barred patterns of length 4}

It is well known that for patterns with 0 bars, permutation patterns fall into the three classes of $\left|S_{n}(\{1234\})\right|,\left|S_{n}(\{1342\})\right|$, and $\left|S_{n}(\{1324\})\right|$, [1]. For the first of these, we have a closed form enumeration, for the second a generating function, and for the third a recurrence that allows enumeration up to $n=20$ [1] [7].

As given by the Lemmas, we need only consider the case of 2 bars and 1 bar in turn.

For two bars, we have two cases: either the forbidden pattern contains a symmetry of a pair of consecutive numbers of the form $(c-1) \bar{c}$, or it does not.

If the forbidden pattern $q$ contains a consecutive $(c-1) \bar{c}$ pattern (or equivalently a consecutive $c \overline{(c-1)}, \overline{(c-1)} c$, or $\bar{c}(c-1)$ pattern), then by Lemma 2 it is equivalent to the pattern $q$. So we need only consider the cases where this does not happen. They are the patterns $\overline{12} 43, \overline{1} 32 \overline{4}$ and their symmetries. However know $\left|S_{n}(\{\overline{12} 43\})\right|=\left|S_{n}(\{\overline{1} 32 \overline{4}\})\right|=$ $(n-2)$ ! for all $n \geqslant 0$ by Lemma 3 ,

Finally, we consider the case of barred patterns of length 4 with precisely one bar. This was first comprehensively studied by Callan 3. The following propositions are proved in a similar way to Callan's work, but with slightly modified notation, and are included for completeness.

Callan showed that permutations avoiding a barred pattern of length 4 with exactly one bar fall into 4 categories. By Lemma 2, 64 of these $96(=4 ! \times 4)$ patterns are equivalent to avoiding an unbarred pattern of length 3 , thus yielding the Catalan numbers. The remaining 3 cases are those for which the sequence $\left\{\left|S_{n}(\{q\})\right|\right\}_{n \geqslant 0}$ gives the Bell numbers, OEIS Sequence A051295, and OEIS Sequence A137533 9]. The data in Table 1] first computed by Callan [3, lists the 32 remaining patterns, grouped by Wilf equivalence class.

\begin{tabular}{|c|c|c|}
\hline Representative & Other Class Members & Sequence \\
\hline $1 \overline{4} 23$ & $134 \overline{2}, 23 \overline{1} 4, \overline{2} 431, \overline{3} 124,32 \overline{4} 1,4 \overline{1} 32,421 \overline{3}$ & Bell \\
\hline$\overline{2} 413$ & $2 \overline{4} 13,24 \overline{1} 3,241 \overline{3}, \overline{3} 142,3 \overline{1} 42,31 \overline{4} 2,314 \overline{2}$ & Bell \\
\hline$\overline{1} 423$ & $\overline{1} 342,241 \overline{4}, 243 \overline{1}, 312 \overline{4}, 324 \overline{1}, \overline{4} 132, \overline{4} 213$ & A 051295 \\
\hline$\overline{1} 324$ & $132 \overline{4}, 423 \overline{1}, \overline{4} 231$ & A 051295 \\
\hline$\overline{1} 432$ & $234 \overline{1}, 321 \overline{4}, \overline{4} 123$ & new \\
\hline
\end{tabular}

Table 1: Permutation Classes Avoiding a Pattern of Length 4 with 1 Bar

We consider one representative from each of these classes. Permutations that avoid other patterns but yield the same counting sequence can be enumerated by similar methods.

Proposition 1. $\left|S_{n}(\{1 \overline{4} 23\})\right|$ satisfies the recurrence

$$
\left|S_{n}(\{1 \overline{4} 23\})\right|=\sum_{i=1}^{n}\left(\begin{array}{c}
n-1 \\
i-1
\end{array}\right)\left|S_{n-i}(\{1 \overline{4} 23\})\right|
$$


Proof. Let $i$ be the position of the letter $n$ in a $1 \overline{4} 23$-avoiding permutation. Then, the $i-1$ letters preceding $n$ must be in decreasing order (otherwise $j<k<n$ forms a 123 pattern without a larger element between the $j$ and $k$ ). The $n-k$ letters after $n$ may be in any order, so long as they avoid $1 \overline{4} 23$. This gives a typical graph of a $1 \overline{4} 23$-avoiding permutation, considered as a function from $[n]$ to $[n]$ as in Figure 1 .

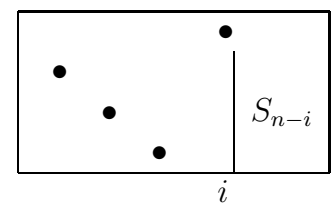

Figure 1: A generic $\{1 \overline{4} 23\}$-avoiding permutation

There are $\left(\begin{array}{c}n-1 \\ i-1\end{array}\right)$ ways to choose the initial $k$ elements, and $\left|S_{n-i}(\{1 \overline{4} 23\})\right|$ ways to order the last $n-i$ elements, so summing over all possible positions $i$ for the entry $n$, we obtain the above recurrence.

This is the same recurrence satisfied by the Bell numbers. Further, this proof gives a clear bijection with set partitions of $\{1, \ldots, n\}$. Given a $\{1 \overline{4} 23\}$-avoiding permutation $p$ let the set containing $n$ in the corresponding set partition be $n$ and all elements that appear before $n$ in $p$. Since the elements of $p$ that after $n$ have the same recursive $\{1 \overline{4} 23\}$-avoiding structure, the rest of the set partition can be computed similarly.

This proof is not completely new. It can be shown bijectively that $S_{n}(\{1 \overline{4} 23\})=$ $S_{n}(12-3)$, where the dash denotes the generalized permutation pattern $12-3$, and $S_{n}(12-3)$ denotes the set of all permutations of length $n$ that avoid copies of the pattern 123 where the first two numbers in the pattern are adjacent. Notice that not only are the cardinalities of these two sets the same, but the sets themselves are identical. Claesson [5] showed that $\left|S_{n}(12-3)\right|$ is given by the $n$th Bell number. A nonrecursive description of this set is as follows: the set of permutations such that the entries between successive right-to-left maxima as well as entries before $n$ are in decreasing order.

Proposition 2. $\left|S_{n}(\{\overline{1} 423\})\right|$ satisfies the recurrence

$$
\left|S_{n}(\{\overline{1} 423\})\right|=\sum_{i=1}^{n}(n-i) !\left|S_{i-1}(\{\overline{1} 423\})\right|
$$

Proof. Let $i$ be the position of the letter 1 . Then the $(n-i)$ entries following $i$ may appear in any order. However, the letters before the 1 must all be smaller than the letters after the 1 , otherwise $j 1 k$ with $j>k$ forms a 312 pattern without a smaller letter in front of it. The $i-1$ entries preceding $i$ must merely avoid the forbidden pattern $\overline{1} 423$, giving the graph of a typical $\overline{1} 423$-avoiding permutation to be as in Figure 2 .

There are $\left|S_{i-1}(\{\overline{1} 423\})\right|$ ways to order the first $i-1$ elements, and $(n-i)$ ! ways to order the last $n-i$ elements, so summing over all possible positions $i$ for the letter $n$ gives the above recurrence. 


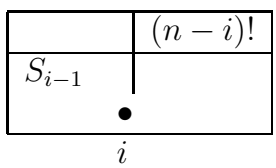

Figure 2: A generic $\{\overline{1} 423\}$-avoiding permutation

This recurrence gives sequence A051295 in the Online Encyclopedia of Integer Sequences.

\section{Proposition 3.}

$$
\left|S_{n}(\{\overline{1} 432\})\right|=(n-1) !+\sum_{j=2}^{n} \frac{(n-2) !}{(j-2) !}+\sum_{i=3}^{n} \sum_{j=2}^{n-i+2} \sum_{l=j+i-2}^{n} \frac{(n-i) !(l-j-1) !}{(l-i) !(i-3) !}
$$

Proof. We break the set $S_{n}(\{\overline{1} 432\})$ into cases depending on the location of the letter 1 .

If 1 is the first letter of a permutation $p$, then clearly $p \in S_{n}(\{\overline{1} 432\})$ since 1 as the first letter cannot be involved in a forbidden 321 pattern, and every 321 pattern is preceded by the 1 . Thus, there are $(n-1)$ ! permutations avoiding $\overline{1} 432$ and beginning with 1 .

If 1 is the second letter of a permutation $p$ that begins with $j$, then we get the following graph:

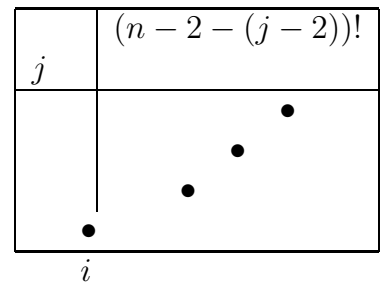

Figure 3: A $\{\overline{1} 432\}$-avoiding permutation with 1 as the second letter

That is, all letters smaller than $j$ must appear in increasing order (otherwise $j>a>b$ forms a 321 pattern without a smaller letter in front of it), so we may choose the positions of these letters but not their order. This can be done in $\left(\begin{array}{c}n-2 \\ j-2\end{array}\right)$ ways. Further, the letters greater than $j$ may appear in any order, but their positions are exactly the positions left over after choosing the places of the letters smaller than $j$. These larger letters can be ordered in $(n-2-(j-2))$ ! ways. Summing over all possible values for $j$, we get the second term in the proposition.

Finally, we consider the case of 1 appearing in the third position or later. We obtain a permutation graph as in Figure 4.

That is, all letters before 1 must appear in increasing order, otherwise $a>b>1$ is a 321 pattern not preceded by a smaller letter. If $j$ is the smallest letter before 1 and $l$ is the largest letter before 1 , we may also conclude that 


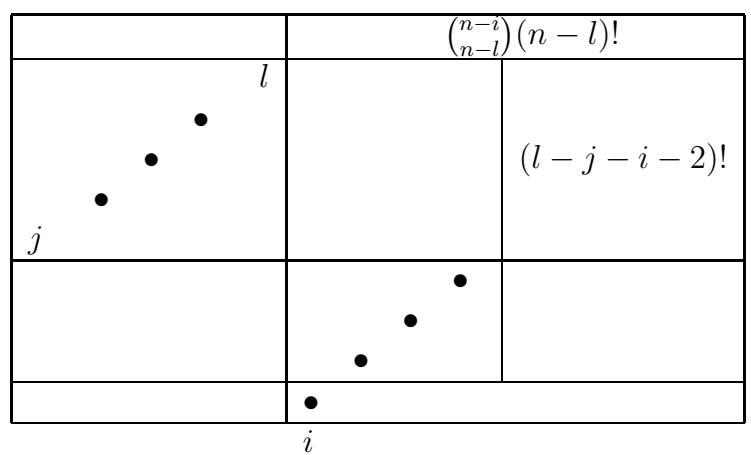

Figure 4: A $\{\overline{1} 432\}$-avoiding permutation with 1 as the third letter or later

- The $n-l$ letters larger than $l$ may appear in any order, so we may choose their positions in $\left(\begin{array}{l}n-i \\ n-l\end{array}\right)$ ways, and their order in $(n-l)$ ! ways.

- The letters smaller than $j$ must appear in increasing order, otherwise $j>a>b$ is a 321 pattern not preceded by a smaller letter.

- The letters smaller than $j$ must appear strictly before the letters between $j$ and $l$ that are after the 1 . Otherwise, let $a$ be a letter $j<a<l$ that occurs before letter $b$, with $b<j$. Then $l a b$ is a 321 pattern not preceded by a smaller letter.

- Now, the positions of the remaining $(l-j-i-2)$ letters is determined, and they can be ordered in $(l-j-i-2)$ ! ways.

Thus, summing over all possibilities for $j, l$, and $i$ gives the third and final term in the proposition.

This formula produces sequence A137533 in the Online Encyclopedia of Integer Sequences.

\subsection{Avoiding barred patterns of length 5}

A comprehensive study of permutations avoiding patterns of length 5 is not yet completed, however, computational data shows that a number of new non-degenerate cases remain to be studied. We give a survey of computational data for $n \leqslant 7$ and patterns with 1,2 , or 3 bars.

The symmetries of reverse, complement, and inverse give 89 distinct equivalence classes for the sequence $\left|S_{n}(\{q\})\right|$ when $q$ is a pattern of length 5 with one bar. Of these classes, 52 are equivalent to avoiding a pattern of length 4 by Proposition 2 .

For the 37 remaining classes, computation suggests that there are at least 17 different possible sequences for $\left|S_{n}(\{q\})\right|$. 15 of these are new to the literature. Table 2 below sorts these non-degenerate results by their first 7 terms.

Similarly, for patterns of length 5 with 2 bars, there are 172 equivalence classes and 150 of these reduce to avoiding an unbarred pattern of length 3 . Of the 22 non-degenerate 


\begin{tabular}{|l|l|l|}
\hline $\begin{array}{l}\text { Pattern Class } \\
\text { Representatives }\end{array}$ & Sequence & OEIS Number \\
\hline $25 \overline{3} 14,35 \overline{2} 41,45 \overline{3} 12,51 \overline{4} 23$ & $1,2,6,23,104,530,2958$ & A117106 \\
\hline $352 \overline{4} 1$ & $1,2,6,23,104,530,2959$ & A137534 (new) \\
\hline $1 \overline{4} 235,4 \overline{2} 513$ & $1,2,6,23,104,531,2977$ & A137535 (new) \\
\hline $423 \overline{1} 5,425 \overline{1} 3,5314 \overline{2}\left(^{* *}\right)$ & $1,2,6,23,104,531,2982$ & A110447 \\
\hline$\overline{4} 2153,5 \overline{1} 423$ & $1,2,6,23,104,532,3002$ & A137536 (new) \\
\hline $5134 \overline{2}$ & $1,2,6,23,104,532,3003$ & A137537 (new) \\
\hline $253 \overline{1} 4, \overline{3} 1542\left(^{*}\right), \overline{3} 5214\left(^{*}\right), \overline{3} 5241$ & $1,2,6,23,104,532,3004$ & A137538 (new) \\
$42 \overline{5} 13,43 \overline{5} 21\left(^{*}\right), 45 \overline{1} 32\left(^{*}\right)$ & & \\
\hline $1 \overline{5} 324, \overline{4} 1523$ & $1,2,6,23,104,532,3005$ & A137539 (new) \\
\hline$\overline{4} 1253$ & $1,2,6,23,104,533,3026$ & A137540 (new) \\
\hline $1 \overline{5} 234,412 \overline{5} 3$ & $1,2,6,23,104,533,3027$ & A137541 (new) \\
\hline$\overline{1} 3425,3524 \overline{1}$ & $1,2,6,23,104,533,3038$ & A137542 (new) \\
\hline$\overline{1} 3245,3241 \overline{5}, \overline{5} 1432, \overline{5} 3412$ & $1,2,6,23,104,534,3060$ & A137543 (new) \\
\hline$\overline{5} 1342$ & $1,2,6,23,104,534,3064$ & A137544 (new) \\
\hline$\overline{5} 2143$ & $1,2,6,23,104,535,3081$ & A137545 (new) \\
\hline $5234 \overline{1}$ & $1,2,6,23,104,535,3082$ & A137546 (new) \\
\hline$\overline{5} 1243$ & $1,2,6,23,104,535,3085$ & A137547 (new) \\
\hline$\overline{5} 1234, \overline{5} 1324$ & $1,2,6,23,104,535,3088$ & A137548 (new) \\
\hline
\end{tabular}

$\left(^{*}\right)$ This sequence will be proven by the method of prefix enumeration schemes.

$(* *)$ This sequence has been proven by Callan in [4].

Table 2: Number of permutations avoiding a pattern of length 5 with one bar

cases, we get at least 13 distinct sequences, 9 of these new to the literature. These sequences are given in Table 3 .

Finally, for patterns of length 5 with 3 bars, all cases are degenerate to either $S_{n}(\{q\})=$ 1 or $S_{n}(\{q\})=(n-3)$ !.

Now that we have exhausted comprehensive case by case analysis of permutations avoiding a single barred permutation, we consider a method to compute recurrences for $S_{n}(Q)$ where $Q$ is an arbitrary set of barred permutation patterns.

\section{Enumeration Schemes}

Our goal in this section is to introduce a single method that works to enumerate many classes $S_{n}(Q)$ where $Q$ is a set of barred permutation patterns. Following Zeilberger 13] [14 and Vatter [10] we derive an algorithm whose input is a set of permutation patterns $Q$, and whose output can be read as a recurrence counting $S_{n}(Q)$. Notation from the Zeilberger's and Vatter's original work with unbarred permutation patterns will be adapted as necessary. 


\begin{tabular}{|c|c|c|}
\hline Pattern Class Representatives & Sequence & OEIS Number \\
\hline $2 \overline{5} 3 \overline{1} 4, \overline{3} 5 \overline{1} 42$ & $1,2,5,14,43,143,509$ & A006789 \\
\hline$\overline{4} 25 \overline{1} 3,5 \overline{1} 32 \overline{4}$ & $1,2,5,14,43,143,510$ & A098569 \\
\hline $145 \overline{32}(*)$ & $1,2,5,14,43,143,511$ & A137549 (new) \\
\hline $251 \overline{43}(*), 415 \overline{32}$ & $1,2,5,14,43,144,522$ & A137550 (new) \\
\hline$\overline{31542}$ & $1,2,5,14,43,144,523$ & A047970 \\
\hline$\overline{2} 413 \overline{5}, 425 \overline{31}, 42 \overline{5} 3 \overline{1}$ & $1,2,5,14,43,144,525$ & A137551 (new) \\
\hline$\overline{1} 43 \overline{5} 2$ & $1,2,5,14,43,145,538$ & A122993 \\
\hline$\overline{1} 5 \overline{2} 43$ & $1,2,5,14,43,146,550$ & A137552 (new) \\
\hline$\overline{21453,243 \overline{15}, 423 \overline{15}, 534 \overline{21}(*)}$ & $1,2,5,14,43,146,561$ & A137553 (new) \\
\hline $354 \overline{21}(*), \overline{5} 324 \overline{1}$ & $1,2,5,14,43,147,575$ & A137554 (new) \\
\hline$\overline{45123}$ & $1,2,5,14,43,147,578$ & A137555 (new) \\
\hline$\overline{1} 432 \overline{5}$ & $1,2,5,14,43,148,592$ & A137556 (new) \\
\hline $345 \overline{21}$ & $1,2,5,14,43,150,617$ & A137557 (new) \\
\hline
\end{tabular}

$\left(^{*}\right)$ This sequence will be proven by the method of prefix enumeration schemes later in this chapter.

Table 3: Number of permutations avoiding a pattern of length 5 with two bars

In the following sections, we discuss in turn the notions of refinement, reversibly deletable elements, gap vectors, and stop points. These four concepts are combined to form an enumeration scheme, or recurrence counting $\left|S_{n}(Q)\right|$.

\section{$3.1 \quad$ Refinement}

Since the set $S_{n}(Q)$ may be complicated, we first partition $S_{n}(Q)$ into disjoint subsets and look for recurrences between these subsets.

One natural and useful way to partition the permutations of $S_{n}(Q)$ is by the patterns formed by the first $i$ letters of its elements. The following notation will be useful to discuss this partitioning of $S_{n}(Q)$ :

$$
\begin{gathered}
S_{n}\left(Q ; p_{1} \cdots p_{i}\right)=\left\{\pi \in S_{n} \mid \pi \text { avoids } q \text { for all } q \in Q, \pi_{1} \cdots \pi_{i} \text { reduces to } p_{1} \ldots p_{i}\right\} \\
S_{n}\left(Q ; \begin{array}{l}
p_{1} \cdots p_{i} \\
l_{1} \cdots l_{i}
\end{array}\right)=\left\{\begin{array}{ll}
\pi \in S_{n} & \begin{array}{l}
\pi \text { avoids } Q \\
\pi_{1} \cdots \pi_{i} \text { reduces to } p_{1} \ldots p_{i}, \text { and } \\
l_{1}, \ldots, l_{i} \text { are the first } i \text { letters of } \pi
\end{array}
\end{array}\right\} .
\end{gathered}
$$

For example, $S_{3}(\{132\} ; 12)=\{123,231\}$, i.e. of the 5 permutations of length 3 that avoid the pattern 132, only 123 and 231 begin with an increasing pair of letters. Similarly, $S_{3}\left(\{132\} ; \begin{array}{c}12 \\ 23\end{array}\right)=\{231\}$. 
Given $p=p_{1} \cdots p_{i}$, a refinement of $p$ is a permutation $q=q_{1} \cdots q_{i+1}$ such that $q_{1} \cdots q_{i}$ reduces to $p$. For example, the refinements of $\emptyset$ are $\{1\}$. The refinements of 1 are $\{12,21\}$. The refinements of 12 are $\{123,132,231\}$.

Finally, we have the following useful observation:

\section{Proposition 4.}

$$
\begin{gathered}
S_{n}(Q ; p)=\bigcup_{q \in \text { refinements of } p} S_{n}(Q ; q) \text {, and so } \\
\left|S_{n}(Q ; p)\right|=\sum_{q \in \text { refinements of } p}\left|S_{n}(Q ; q)\right| .
\end{gathered}
$$

Thus, for any set of patterns $Q$, we have $S_{n}(Q)=S_{n}(Q ; 1)=S_{n}(Q ; 12) \cup S_{n}(Q ; 21)=$ $\cdots$. This partitioning of $S_{n}(Q)$ into disjoint sets depending on the initial few letters is identical to the work of Zeilberger.

Graphically, we may represent refinement using a graph, where the vertices correspond to the sets $S_{n}(Q ; p)$ and are labelled with the prefixes $p$. There is a directed edge from a prefix to each of its refinements. To count $S_{n}(Q)$ it is enough to count the subsets $S_{n}(Q ; p)$ represented by the leaves of the graph. An example of such a graph of refinements is given in Figure 5 .

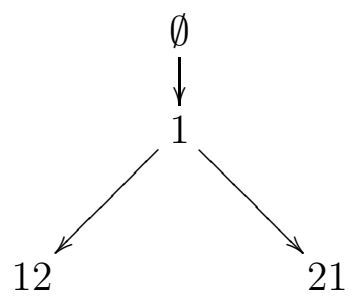

Figure 5: The graph of refinements for an arbitrary pattern set

Now that we have a way to partition $S_{n}(Q)$ into disjoint subsets, we consider ways to find recurrences between these subsets.

\subsection{Reversibly Deletable Elements}

The key tool for finding recurrences between $S_{n}(Q ; p)$ for various prefixes $p$ is the following:

Definition 1. Given $Q$, a set of barred permutation patterns, and $p$, a prefix of length $l, l>0$, we say that position $r(1 \leqslant r \leqslant l)$ is reversibly deletable if and only if the action of removing $p_{r}$ from a $Q$-avoiding permutation of length $n$ and inserting $p_{r}$ into a $Q$-avoiding permutation of length $n-1$ is a bijection between $S_{n}\left(Q ; \begin{array}{l}p_{1} \cdots p_{l} \\ i_{1} \cdots i_{l}\end{array}\right)$ and $S_{n-1}\left(Q ; \begin{array}{l}p_{1} \cdots p_{r-1} p_{r+1} \cdots p_{l} \\ i_{1} \cdots i_{r-1} i_{r+1} \cdots i_{l}\end{array}\right)$ 
In the case of unbarred pattern avoidance, it is enough to check that the insertion of $p_{r}$ into a $Q$-avoiding permutation of length $n-1$ gives a $Q$-avoiding permutation of length $n$, since the deletion of a letter from a permutation cannot cause a bad pattern. More specifically, for unbarred pattern avoidance, $p_{r}$ is reversibly deletable if and only if every forbidden pattern involving $p_{r}$ implies the existence of a forbidden pattern without $p_{r}$.

For example, if $Q=\{123\}$, and $p=21$, we have that $p_{1}=$ "2" is reversibly deletable, since the only way for $p_{1}$ to be involved in a 123 pattern is for there to be $21 \cdots p_{s} \cdots p_{t}$ with " 2 " $<p_{s}<p_{t}$. But this means that " 1 " $<p_{s}<p_{t}$, and $1 p_{s} p_{t}$ forms a forbidden 123 pattern without $p_{1}=$ " 2 ". That is, every 123 pattern involving $p_{1}$ implies the existence of a 123 pattern without $p_{1}$. Thus, it is impossible to create a $\{123\}$-containing permutation by inserting $p_{1}$ into a $\{123\}$-avoiding permutation. So inserting and deleting $p_{1}$ is indeed a bijection between $\{123\}$-avoiding permutations of length $n-1$ and $\{123\}$-avoiding permutations of length $n$.

For the case of barred patterns, the definition of reversibly deletable elements is equivalent to the old definition with the added caveat that $p_{r}$ cannot be the only letter to play the role of a barred letter in extending a forbidden $q$ pattern to $\bar{q}$. That is, deleting $p_{r}$ from a $Q$-avoiding permutation can only fail to produce another $Q$-avoiding permutation if $p_{r}$ plays the role of a barred letter and its removal makes an instance of a forbidden pattern $q$ no longer extendable to the larger barred pattern $\bar{q}$.

In summary, to check algorithmically that $p_{r}$ is reversibly deletable, we must check two things.

1. Check that inserting $p_{r}$ into a $Q$-avoiding permutation always produces a $Q$-avoiding permutation.

2. Check that deleting $p_{r}$ from a $Q$-avoiding permutation always produces a $Q$-avoiding permutation.

We discuss how to rigorously check each of these in turn.

\section{Insertion}

For insertion, as in the unbarred case, we check that every possible occurrence of a forbidden pattern with $p_{r}$ implies the existence of a forbidden pattern without $p_{r}$. This is easily seen to happen in a finite number of scenarios. First, choose the letters of the prefix $p$ (including $p_{r}$ ) that will be involved in the forbidden pattern. Then, choose all the ways that the remaining letters of the forbidden pattern can be spaced between the letters of $p$.

For example, for $Q=\{\overline{1} 423\}$, a forbidden pattern is a 312 pattern without a smaller letter before it. Consider $p=123$, and reversibly deletable candidate $p_{2}=$ "2" . Recall that $p$ is a prefix, denoting that the first three letters of our permutation are increasing, not that they are specifically the letters 1,2 , and 3 . So the only way for $p_{2}$ to be involved in a bad pattern is for $p_{2}$ to be followed by a smaller increasing pair. These letters may be (a) both less than $p_{1}$, (b) one less than $p_{1}$ and one greater than $p_{1}$, or (c) both greater than $p_{1}$ and less than $p_{2}$, as in the permutation 
graphs in Figure 6 below. We consider a permutation as a function from $\{1, \ldots, n\}$ to $\{1, \ldots, n\}$. We use $*$ to mark $p_{2}$ to be deleted, $\mathbf{\square}$ to denote the letters of $123 a b$ that, together with $p_{2}$, form a forbidden pattern, and $\square$ to mark another letter that, together with the letters marked $\mathbf{\square}$, forms a forbidden pattern without $p_{2}$.

We quickly eliminate case (c) since $123 a b$ where $2 a b$ is a 312 pattern, and "1" $<a<$ $b<$ "2", actually extends to the 1423 pattern $12 a b$. Now, it is easy to check that deleting $p_{2}$ in each of cases (a) and (b) gives another $\{\overline{1} 423\}$-containing permutation.

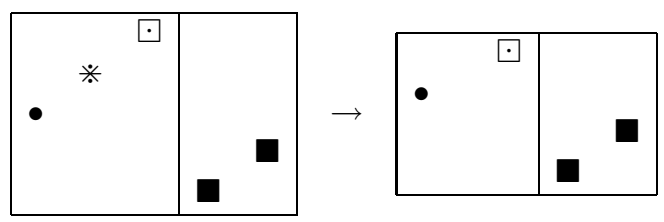

Case (a): both post-prefix letters less than $p_{1}$

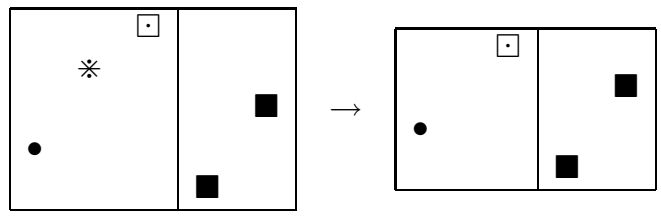

Case (b): one post-prefix letter less than $p_{1}$ and one greater than $p_{1}$

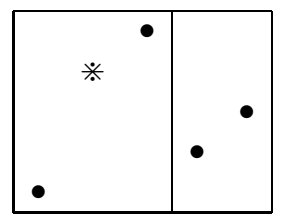

Case (c): both post-prefix letters greater than $p_{1}$ and less than $p_{2}$

Figure 6: An example of checking that insertion is bijective

Additionally, to check that $p_{r}$ is reversibly deletable for prefix $p$ and forbidden pattern $q$ where $q$ has $b$ bars, we must also check scenarios with $b$ additional letters.

These additional scenarios are indeed necessary. For example, let $Q=\{134 \overline{2}\}$, and $p=21$, and consider $p_{1} . p_{1}$ being involved in a bad pattern with two letters after the prefix may happen in one way, namely:

So, $21 a b$ containing the forbidden pattern " 2 " $<a<b$ does imply that "1" $<a<b$ is a forbidden pattern without $p_{1}$. It seems from this that $p_{1}$ is reversibly deletable. However, 21abc containing the forbidden pattern $2 a b$ does not imply that $1 a b c$ is bad (if "1" $<c<$ "2"), since $c$ may act as a barred letter extending the $1 a b$ pattern, but not the $2 a b$ pattern. 


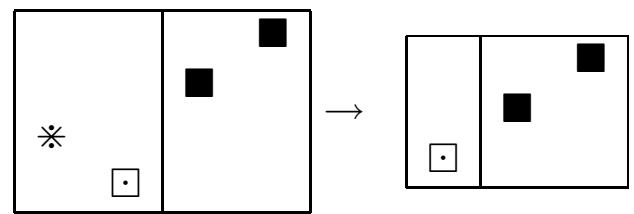

Figure 7: A $\{134 \overline{2}\}$-avoiding permutation with prefix 21

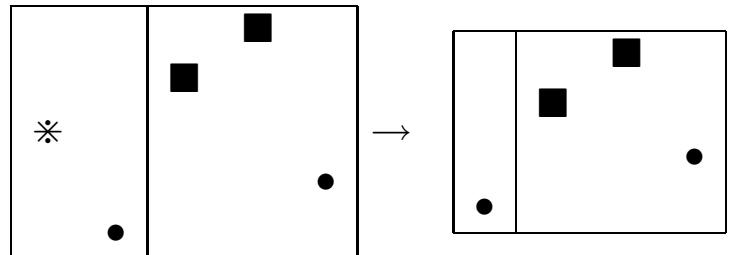

Figure 8: A $\{134 \overline{2}\}$-containing permutation with prefix 21

To show that this is always enough, note that if $\pi$ contains a forbidden pattern $\underline{q}$ where $q$ has $b$ bars, then only $b$ letters need be inserted to form a copy of the $\bar{q}$, so adding even more letters is redundant.

\section{Deletion}

Now that we have rigorously shown that insertion of $p_{r}$ is a map from $Q$-avoiding permutations to $Q$-avoiding permutations, we check that deletion also has this property.

To do this, we check the scenarios for a forbidden pattern involving $p_{r}$ as above. Namely, we want to show that if $\pi^{*}$ begins with prefix $p^{*}=p_{1} \cdots p_{r-1} p_{r+1} \cdots p_{l}$ and has a forbidden pattern, then $\pi$, beginning with $p=p_{1} \cdots p_{r} \cdots p_{l}$ has a forbidden pattern. Thus, if we compute all the scenarios beginning with $p^{*}$, insert $p_{r}$, and check that each one contains a forbidden pattern, then we are done.

For example, if $Q=\{\overline{1} 423\}$ and $p=123$, we again consider $p_{2}$. There are a number of ways for $p_{1} p_{3}$ to be involved in a forbidden pattern that does not extend to 1423 , namely:
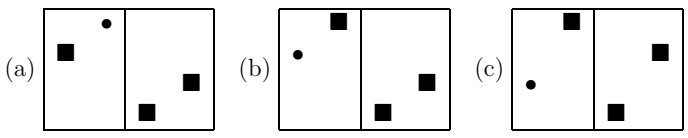

Figure 9: $\{\overline{1} 423\}$-containing patterns with prefix 12 .

Now, $p_{2}$ can be inserted into each of these scenarios in possibly multiple ways as in Figure 10.

We may inspect that each of these resulting permutations contains a 312 pattern that does not extend to a 1423 pattern, and thus, $p_{2}$ is reversibly deletable. 


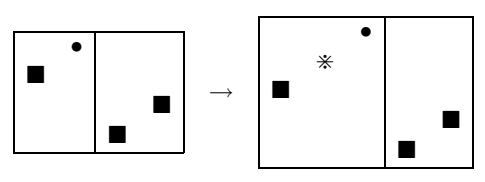

Case (a): inserting $p_{2}\left(p_{1}<p_{2}<p_{3}\right)$ into a $\{\overline{1} 423\}$-avoiding permutation

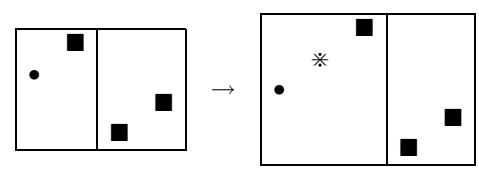

Case (b): inserting $p_{2}\left(p_{1}<p_{2}<p_{3}\right)$ into a $\{\overline{1} 423\}$-avoiding permutation

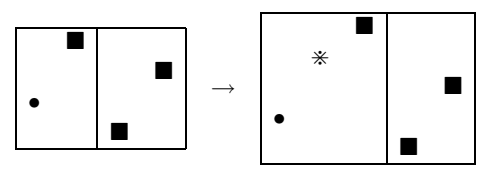

Case (c1): inserting $p_{2}\left(p_{1}<p_{2}<p_{3}\right)$ into a $\{\overline{1} 423\}$-avoiding permutation

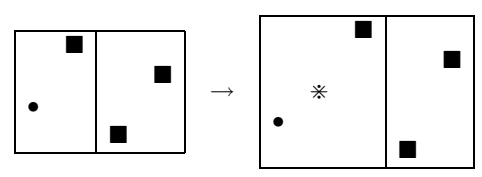

Case (c2): inserting $p_{2}\left(p_{1}<p_{2}<p_{3}\right)$ into a $\{\overline{1} 423\}$-avoiding permutation

Figure 10: An example of checking that deletion is bijective

To denote that $p_{r}$ is reversibly deletable in the graphical notation, we draw a dotted arrow from $p$ to $p^{*}$ labelled with $d_{r}$, which denotes the deletion map of deleting the $r$ th letter of $\pi$ and reducing. For example, if $p=21$ had $p_{1}$ reversibly deletable, we would encode this as in Figure 11.

\subsection{Gap Vectors}

Unfortunately, the reversibly deletable elements are usually not sufficient to find recurrences counting the elements of $S_{n}(Q)$, so following Vatter [10], we introduce the notion of gap vectors. Given a set of forbidden patterns $Q$ and prefix $p=p_{1} \cdots p_{i}$, a spacing vector $v$ is a vector in $\mathbb{N}^{i+1}$. We write $\|v\|$ to denote the sum of the entries of $v$. Spacing vectors help further narrow down the set $S_{n}(Q ; p)$ into smaller subsets in the following way.

Definition 2. Given $Q$, a set of forbidden patterns, $p$ a prefix of length $i$, and $v$, a spacing vector of length $l+1$, let $s_{1} \cdots s_{l}$ be the permutation obtained by sorting $p . S_{n}(Q ; p ; v)$ denotes the set of permutations of length $n$, avoiding $Q$, beginning with prefix $p$, and with 


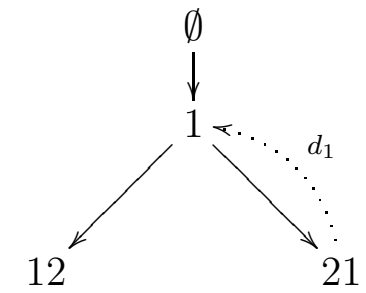

Figure 11: Representation of reversibly deletable elements

exactly $v_{1}$ letters smaller than $s_{1}$, exactly $v_{j}$ letters that are greater than $s_{j-1}$ and smaller than $s_{j}$, and exactly $v_{i+1}$ letters that are greater then $s_{l}$.

For example, $S_{n}(\{123\} ; 12 ;\langle 0,1,2\rangle)$ denotes the set of permutations avoiding 123 , beginning with an increasing pair of letters $p_{1} p_{2}$, with one letter $a$ such that $p_{1}<a<p_{2}$ and two letters bigger then $p_{2}$.

Definition 3. A spacing vector $v$ is a gap vector for $[Q, p]$ if there are no permutations avoiding $Q$ with prefix $p$ and spacing vector $\geqslant v$ (componentwise).

For example, if $Q=\{123\}$ and $p=12$, then $v=\langle 0,0,1\rangle$ is a gap vector since if we have at least one letter $a$ larger than $p_{1}$ and $p_{2}$, then $p_{1} p_{2} a$ forms a 123 pattern.

We check this similarly to the unbarred case, with yet another constraint.

To check that $v$ is a gap vector in the unbarred case, we consider permutations starting with $p$, and name $v_{1}$ letters smaller than $s_{1}=1$ (say $\left.\frac{1}{v_{1}+1}, \ldots, \frac{v_{1}}{v_{1}+1}\right), v_{2}$ letters between $s_{1}=1$ and $s_{2}=2\left(\right.$ say $\left.1+\frac{1}{v_{2}+1}, \ldots 1+\frac{v_{2}}{v_{2}+1}\right), \ldots$, and $v_{l+1}$ letters bigger than $s_{l}=l$ (say $\left.l+\frac{1}{v_{l+1}+1}, \ldots, l+\frac{v_{l+1}}{v_{l+1}+1}\right)$. Now, consider all permutations that begin with $p$ and end with any of the $\left(v_{1}+\cdots+v_{l+1}\right)$ ! permutations of these fractional letters. If each of these permutations contains a forbidden pattern from $Q$, then $v$ is a gap vector for $[Q, p]$.

In the barred case, while the algorithm in the previous paragraph is necessary to show that $v$ is a gap vector, it is no longer sufficient. For example, when avoiding $Q=\{23 \overline{1}\}$, with prefix $p=1, v=\langle 0,1\rangle$ appears to be a gap vector when considering permutations of length 2. However, there are permutations of length 3 with spacing vector $v^{*}=\langle 1,1\rangle$ that avoid $Q$. That is, we may have a vector such that $\left|S_{n}(Q ; p ; v)\right|=0$, but there is some $w>v$ (componentwise) such that $\left|S_{n}(Q ; p ; w)\right|>0$. However, we want to find a basis for the set of vectors $v$ such that $\left|S_{n}(Q ; p ; w)\right|=0$ for all $n$ and for all $w \geqslant v$.

In light of this complication, to show that $v$ is a gap vector for $[Q, p]$, not only do we need to confirm that there are no $Q$-avoiding permutations with prefix $p$ and spacing $v$, but also that there are no $Q$-avoiding permutations with spacing $w, w>v$ componentwise. More precisely, if we are concerned with finding the basis of gap vectors for $[Q, p]$, most of the time we proceed as in the unbarred case, with one important exception. More work needs to be done to check for gap vectors when avoiding a pattern of the form $q=q_{1} \cdots q_{m-i-1} \bar{q}_{m-i} \cdots \bar{q}_{m}$. We begin with the case when $i=0$, i.e. $q$ ends with exactly one barred letter. 
Theorem 1. Let $q \in \bar{S}_{m}$ such that $\underline{q}=q_{1} \cdots q_{m-1}$. Then there are no basis gap vectors for $[\{q\}, p]$ for any prefix $p$.

Proof. Assume that $q$ is as in the proposition, and that $v$ is a basis gap vector for $[\{q\}, p]$. Now, let $\pi=\pi_{1} \cdots \pi_{l} \in S_{|p|+\|v\|}$ that has prefix $p$. Since $v$ is a basis gap vector, $\pi$ contains $q$, but if the last letter of $\pi$ is deleted, then it avoids $q$. That is, by definition of basis gap vector, the last letter of $\pi$ is involved in a forbidden $\underline{q}$ pattern that does not extend to a $\bar{q}$ pattern.

For each instance of $q$ in $\pi$, choose a letter to append to $\pi$ which will extend the instance to $\bar{q}$; write $L$ for the set of such letters to be appended to $\pi$. Without loss of generality, assume that $q_{m-1}<q_{m}$. Then append the letters of $L$ to $\pi$ in increasing order, and call the resulting permutations $\pi^{*}$. We claim that either $\pi^{*}$ is a $\{q\}$-avoiding permutation or can be further extended to be $\{q\}$-avoiding, with prefix $p$ and spacing $w$, $w>v$, so $v$ is not a gap vector, and by contradiction we are done.

To see that $\pi^{*}=\pi_{1}^{*} \cdots \pi_{l_{2}}^{*}$ is $\{q\}$-avoiding, we consider several cases.

First, by Lemma 2, we may assume that $\bar{q}$ is not a monotone pattern and that there exists $q_{c}$ with $q_{m-1}<q_{c}<q_{m}$.

Construct $\pi^{*}$ by appending each letter of $L$ individually. Suppose that $\pi_{1} \cdots \pi_{l+i}$ contains a forbidden $\underline{q}$ pattern that uses $\pi_{l+i}$. Then either:

- The rest of the forbidden pattern consists of letters $\pi_{j}$ with $j \leqslant l$. In this case, the letter of $L$ that was meant to extend the bad pattern formed by replacing $\pi_{l+i}$ with $\pi_{l}$ has yet to be appended to $\pi$, and will extend this instance of $q$ to an instance of $\bar{q}$ as well.

- If the rest of the forbidden pattern consists of both letters from $\pi_{1} \cdots \pi_{l}$ and letters from $L$, then we note that by Lemma 2, there exists $\pi_{c}$ in the instance of the forbidden pattern with $\pi_{l+j}<\pi_{c}<\pi_{l+i}, j<i, c<l$, so we may append another letter $\pi_{l+i+1}$ to $\pi^{*}$ extending this to a copy of $\bar{q}$. Again, there must be $\pi_{c_{2}}$ with $c_{2}<l$ so that $\pi_{l+i}<\pi_{c_{2}}<\pi_{l+i+1}$. If this letter $\pi_{l+i+1}$ is involved in another instance of $\underline{q}$, repeat. We know this process terminates because there are a finite number of letters in $\pi$, so there is a maximum letter of $\left\{\pi_{1}, \ldots, \pi_{l}\right\}$ to play the role of $\pi_{c_{i}}$ in this construction.

In both cases, we have shown that it is possible to append enough letters to $\pi$ to make it $\{q\}$-avoiding, and thus there are no gap vectors for $[\{q\}, p]$.

As an example of this construction, consider permutations that avoid the pattern $q=241 \overline{3}$. For the prefix 123, there are no permutations avoiding $q$ with spacing $\langle 1,0,0,0\rangle$, and there are no permutations avoiding $q$ with a spacing vector of weight 2 ; however, we can construct a permutation with prefix $p=123$ and a spacing vector of weight 3 that avoids $q$. Notice that in the language of the proposition, $\pi=2341$, which contains $\bar{q}=231$ in several places, namely, 231, 241, and 341. Thus, we require the addition of a letter greater than " 2 " and less than " 3 ", a letter greater than " 2 " and less than " 4 ", and a letter greater than " 3 " and less than " 4 ", to extend each of these copies of $\underline{q}$ to a copy of 
$\bar{q}$. Choosing two letters, $a$ and $b$ with " $2 "<a<$ " 3 " $<b<$ " 4 " suffices. We append $a$ and $b$ to the end of $\pi$ to obtain $246135 \in S_{n}(\{241 \overline{3}\} ; 123 ;\langle 1,1,1,0\rangle)$. Note that 246135 is $\{241 \overline{3}\}$-avoiding, with prefix 123 and a spacing vector which is greater than $\langle 1,0,0,0\rangle$.

We note that Theorem 11 is necessary. In general, we can find $p$ and $q$ so that the smallest weight of a spacing vector $v$ where $S_{n}(\{q\} ; p ; v) \neq \emptyset$ is arbitrarily large, and checking all the appropriate scenarios would be time-consuming. With this Theorem, we need not consider all of these scenarios, but rather return that the set of gap vectors for $[\{q\}, p]$ is empty. Since gap vectors were included in the scheme algorithm to help find recurrences, eliminating gap vectors in this case may seem to limit the success of our algorithm. However, we note that via the symmetries of the square, if we cannot find an enumeration scheme for $S_{n}(\{q\})$ where $q$ ends in a barred letter, we may still find an enumeration scheme for $S_{n}\left(\left\{q^{r}\right\}\right)$ where $q^{r}$ does not end in a barred letter. Further, if $q$ is part of a set of forbidden patterns $Q$, the other patterns not ending in a barred letter may still help find gap vectors for the enumeration scheme for $S_{n}(Q)$.

We also note that this construction does not necessarily generalize to patterns of the form $q=q_{1} \cdots q_{m-i-1} \bar{q}_{m-i} \cdots \bar{q}_{m}$ where $i>0$. For example, if $q=351 \overline{42}$ and $p=231$, we note that $v=\langle 0,0,0,0\rangle$ is a gap vector because two letters $a$ and $b$, with $p_{3}<b<$ $p_{1}<a<p_{2}$ must be appended to $p$ to extend $p$ to an instance of 35142. But now, $p_{1} a b$ is a new forbidden 351 pattern that requires two letters $c$ and $d$ to be appended with $b<d<p_{1}<c<a$ to extend $p_{1} a b$ to an instance of 35142. This process continues indefinitely.

For the case of patterns which consist of a block of unbarred letters followed by a block of more than one barred letter, we must check extra scenarios to determine if $v$ is a gap vector. Namely, if $S_{n}(Q ; p ; v)=\emptyset$, we must also check that $S_{n}(Q ; p ; w)=\emptyset$ for all $w>v$ with $\|w\|=\|v\|+($ total number of bars in all patterns in $Q$ ). (total number of occurrences of $q_{1} \cdots q_{m-i-1}$ in $\left.p\right\}$ ) before concluding that $v$ is in fact a gap vector.

With this more specific definition of gap vector, we have an ideal in $\mathbb{N}^{l+1}$ which necessarily has a finite basis. We have also exhibited a method to find basis vectors for a scheme. These serve to narrow down the cases we must consider to decide if an element is reversibly deletable.

Graphically, we write a basis for the gap vectors corresponding to $p$ below $p$. For example, if $\langle 0,0,1\rangle$ is a gap vector for the prefix 12 , and this causes $p_{2}$ to be reversibly deletable, we would represent this situation as in Figure 12

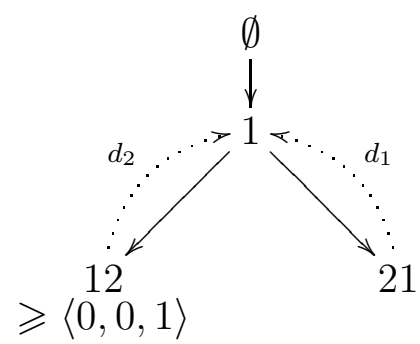

Figure 12: Representation of gap vectors 
A final remark for gap vectors concerns the vector $v_{0}=\langle 0, \ldots, 0\rangle$. Note that if $v_{0}$ is a gap vector for $[Q, p]$, then there are no permutations of any length avoiding $Q$ and beginning with prefix $p$. Since the gap vector $v_{0}$ already indicates that $\left|S_{n}(Q ; p)\right|=0$, it is unnecessary to write $S_{n}(Q ; p)$ in terms of smaller sets. However, for completeness of the definition of enumeration scheme (below), if $v_{0}$ is a gap vector, we allow any one of the $p_{i}$ to be "exceptionally" reversibly deletable. We will return to this remark later.

\subsection{Stop Points}

As we have observed with reversibly deletable elements and with gap vectors, barred patterns require added considerations to find a rigorous enumeration schemes. While we have introduced enough notation to find recurrences between the subsets $S_{n}(Q ; p)$, we require one extra tool to find the base cases for these recurrences, i.e. stop points.

The key observation is that there may be no permutations of length $n$ that avoid $Q$ and begin with prefix $p$, but there may be such permutations of length $n+k$ for some $k>0$. For example, if $Q=\{23 \overline{1}\}$, there are no permutations of length 2 avoiding $Q$, but 231 is a permutation of length 3 , beginning with a 12 pattern. Thus, in the notation of the enumeration scheme, we require a mechanism to indicate at what length we may begin to consider permutations beginning with that prefix.

Definition 4. Given a set of forbidden patterns $Q$, and a prefix $p$ without reversibly deletable elements, we say $s \geqslant|p|$ is a stop point for $[Q, p]$ if there are no permutations of length $\leqslant s$ that avoid $Q$ and begin with prefix $p$

For example, the set of stop points for $(\{23 \overline{1}\}, 12)$ is $\{2\}$.

Proposition 5. Given $Q$ and $p$, the set $S$ of stop points is finite.

Proof. Notice that since $S$ is a set of positive integers, it is enough to show that $S$ has a well defined maximum element.

It is enough to note that stop points are only defined for prefixes with no reversibly deletable elements. If there were no permutations beginning with prefix $p$, we would obtain the gap vector $\langle 0, \ldots, 0\rangle$, and by convention, position 1 is reversibly deletable, so $p$ by definition has an empty set of stop points.

Since $p$ has no reversibly deletable elements, then, we know that there is a permutation $\pi$ of minimal length that begins with $p$ and avoids $Q$. The set of stop points has maximum $|\pi|-1$

The simplest example of a scheme that requires stop points is the scheme for permutations avoiding $\{123,321,23 \overline{1}\}$. Graphically, we represent stop points as a set after an asterisk, listed next to the permutation prefix $p$, as by $p=12$ in the scheme for $S_{n}(\{123,321,23 \overline{1}\})$ in Figure 13

From this scheme, we have

- $\left|S_{0}(Q)\right|=1$ 


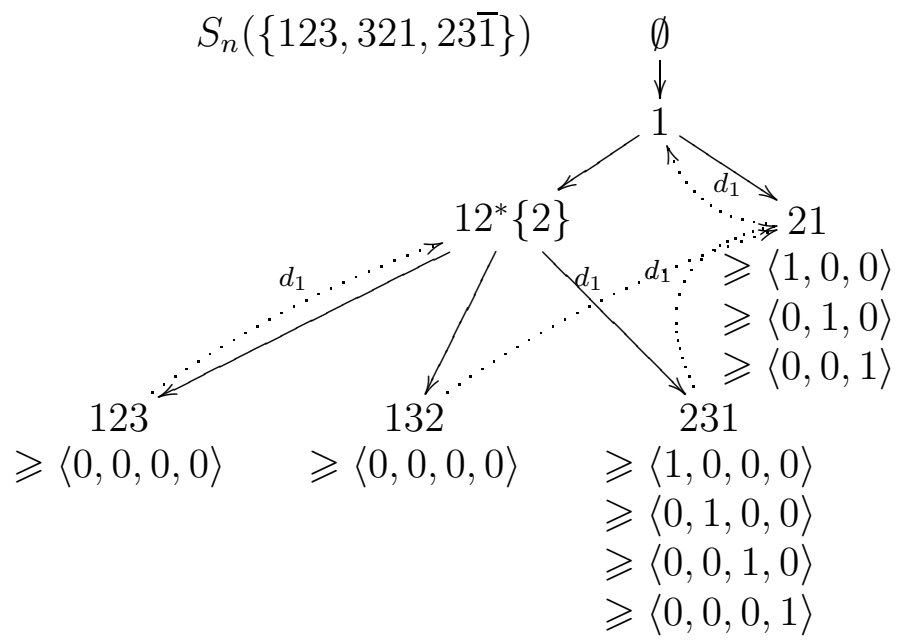

Figure 13: A scheme involving stop points

- $\left|S_{1}(Q)\right|=1$

- $\left|S_{2}(Q)\right|=\left|S_{2}(Q ; 12)\right|+\left|S_{2}(Q ; 21)\right|=0+\left|S_{1}(Q ; 1)\right|=0+1=1$

- $\left|S_{3}(Q)\right|=\left|S_{3}(Q ; 123)\right|+\left|S_{3}(Q ; 132)\right|+\left|S_{3}(Q ; 231)\right|+\left|S_{3}(Q ; 21)\right|$

$=0+0+\left|S_{2}(Q ; 21)\right|+0$

$=0+0+\left|S_{1}(Q ; 1)\right|+0$

$=0+0+1+0=1$

- $\left|S_{n}(Q)\right|=0$ for all $n \geqslant 4$

Without stop points, we would have computed $\left|S_{2}(Q)\right|=2$.

\subsection{Enumeration Schemes}

Finally, we have all the necessary tools to algorithmically find recurrences counting the elements of $S_{n}(Q)$ where $Q$ contains barred permutation patterns. More specifically:

Definition 5. An enumeration scheme $\mathbb{S}$ is a set of 4-tuples $t=\left[p_{j}, R_{j}, G_{j}, S_{j}\right]$ such that for each $t$ :

- $p_{j}$ is a reduced prefix of length $i$.

- $R_{j}$ a (possibly empty) subset of $\{1, \ldots, i\}$.

- $G_{j}$ is a (possibly empty) set of vectors of length $i+1$.

- $S_{j}$ is a (possibly empty) finite set of positive integers whose minimum element is $\geqslant\left|p_{j}\right|$, and

- either $R_{j}$ is non-empty, or all refinements of $p_{j}$ are also in the scheme. 
We have detailed how to find each of the elements of such a 4-tuple, namely if $p_{j}$ is a prefix, denoting the set $S_{n}\left(Q ; p_{j}\right)$, then $R_{j}, G_{j}$, and $S_{j}$ are the corresponding reversibly deletable elements, set of gap vectors, and set of stop points.

The last condition ensures that the enumeration scheme can be read as a recurrence counting the elements of $S_{n}(Q)$. Recall that if $R_{j}$ is non-empty, then we have a bijection between $S_{n}(Q ; p)$ and $S_{n-1}\left(Q ; p^{*}\right)$ for some $p^{*}$. If $R_{j}$ is empty, then we require all refinements of $p_{j}$ to be in the scheme for completeness.

Given an enumeration scheme $\mathbb{S}$ corresponding to pattern set $Q$, we can compute $\left|S_{n}(Q)\right|$ in the following way:

1. Let $P$ be the set of $p_{j}$ such that either (i) $p_{j}$ is a prefix of length $\leqslant n$ with reversibly deletable elements or (ii) $p_{j}$ is a prefix of length $n$ without reversibly deletable elements. We have $\left|S_{n}(Q)\right|=\sum_{p_{j} \in P}\left|S_{n}\left(Q ; p_{j}\right)\right|$

2. For each $p_{j} \in P$, if $n \in S_{j}$, then we have $\left|S_{n}\left(Q ; p_{j}\right)\right|=0$.

3. For each remaining $p_{j} \in P$, associate the set of spacing vectors $v_{j}^{*}$ of all vectors of length $\left|p_{j}\right|+1$ and weight $n-\left|p_{j}\right|$ minus the set of gap vectors $G_{j}$. We have $\left|S_{n}\left(Q ; p_{j}\right)\right|=\sum_{v \in v_{j}^{*}}\left|S_{n}\left(Q ; p_{j} ; v\right)\right|$.

4. For each $p_{j} \in P$, and $v \in v_{j}^{*}$, if $R_{j}$ is non-empty, we have

$$
\left|S_{n}\left(Q ; p_{j} ; v\right)\right|=\left|S_{n-1}\left(Q ; p_{j}^{*}, v^{*}\right)\right|
$$

for prefix $p_{j}^{*}\left(p_{j}\right.$ with letter $r$ deleted) and vector $v^{*}=\left\langle v_{1}, \ldots, v_{r-1}+v_{r+1}, \ldots, v_{n+1}\right\rangle$. If $R_{j}$ is empty, then $\left|S_{n}\left(Q ; p_{j}^{*}\right)\right|=1$.

\section{The Maple Package bVATTER}

The algorithms both (i) to find a scheme and (ii) to read a scheme into a sequence have been programmed in the Maple package bVATTER, available from the author's website: http://faculty.valpo.edu/lpudwell/maple.html. The main functions are SchemeImage, SeqS, Sipur.

SchemeImage inputs a set of patterns $Q$, a maximum depth scheme to search for, and a maximum weight of gap vectors to search for, and outputs a concrete enumeration scheme for words avoiding $Q$ of the specified maximum depth. If it cannot find a scheme for $Q$, it searches for a scheme for a symmetry-equivalent pattern set and returns that scheme instead.

SeqS inputs a scheme and an integer $K$, and uses the scheme to compute $\left|S_{i}(Q)\right|$ for $1 \leqslant i \leqslant K$.

Sipur inputs a list $[L]$ of pairs of integers, a maximum scheme depth, a maximum weight of gap vectors, and an integer $K$. It outputs all information about schemes for permutations avoiding one pattern of each length in $L$ where each pair is of the form [length,number of bars]. For example, Sipur $([4,1]], 4,2,30)$ outputs all information 
about permutations avoiding one pattern of length 4 with 1 bar. It will search for schemes of depth 4 with maximum gap vector weight 2 and will output the first 30 terms of the sequence $\left|S_{i}(Q)\right|$ given by each scheme it finds.

Sipur has been run on $[L]$ for various lists of the form $\left[\left[3, x_{i}\right]^{a},\left[4, y_{i}\right]^{b},\left[5, z_{i}\right]^{c}\right]$, and the output is available from the author's website.

\section{Success Rate}

In this section, we consider the success rate of prefix enumeration schemes for $S_{n}(Q)$ where $Q$ is a set of barred permutation patterns.

Recall that sets of permutation patterns can be put into equivalence classes based on the permutation involutions of reverse, complement and inverse. We measure success in terms of the number of such equivalence classes for which there is an enumeration scheme. In the Table 4, pattern lengths denotes the lengths of patterns, as well as the number of bars. For example pattern lengths $[4,0],[4,1]$ denotes two patterns of length 4 , one without bars, and one with precisely one bar. Specific schemes for the data in the table can be found at the author's website. As for words, it should be noted that pattern sets that are counted as unsuccessful do not necessarily lack an enumeration scheme; they may have enumerations schemes of greater depth than the computer has searched.

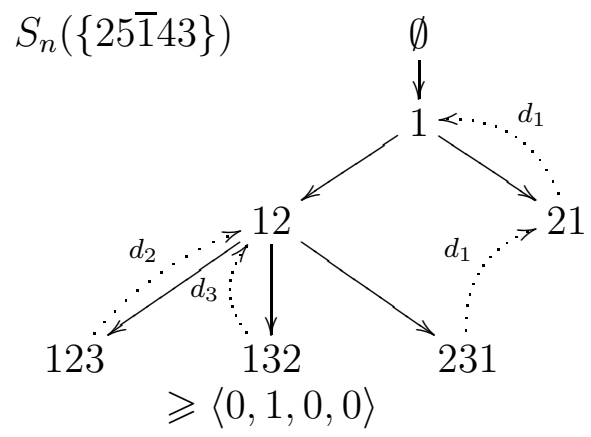

Figure 14: The scheme for $S_{n}(\{25 \overline{1} 43\})$

\subsection{Examples}

We now examine the enumeration schemes for permutations avoiding some specific barred patterns of length 5, thus exhibiting recurrence relations for five of the new sequences given in the tables of Section 2.4.

First, we consider the four classes of patterns of length 5 with one bar that give the sequence $1,2,6,23,104,532,3004$. These are the classes with representatives $25 \overline{1} 43$, $25 \overline{1} 34,43 \overline{5} 21$, and $43 \overline{5} 12$.

For the class represented by $25 \overline{1} 43$ we have the scheme in Figure 14: 


\begin{tabular}{|c|c|c|c|}
\hline Pattern Lengths & Success Rate & Pattern Lengths & Success Rate \\
\hline$[2,0]$ & $1 / 1(100 \%)$ & {$[3,0],[3,0],[3,0]$} & $5 / 5(100 \%)$ \\
\hline$[2,1]$ & $1 / 1(100 \%)$ & {$[3,0],[3,0],[3,1]$} & $43 / 45(95.6 \%)$ \\
\hline$[2,0],[2,0]$ & $1 / 1(100 \%)$ & {$[3,0],[3,0],[3,2]$} & $45 / 45(100 \%)$ \\
\hline$[2,1],[2,0]$ & $2 / 2(100 \%)$ & {$[3,0],[3,1],[3,1]$} & $135 / 138(97.8 \%)$ \\
\hline$[2,1],[2,1]$ & $2 / 2(100 \%)$ & {$[3,0],[3,1],[3,2]$} & $280 / 280(100 \%)$ \\
\hline$[3,0]$ & $2 / 2(100 \%)$ & {$[3,0],[3,2],[3,2]$} & $138 / 138(100 \%)$ \\
\hline$[3,1]$ & $4 / 4(100 \%)$ & {$[3,1],[3,1],[3,1]$} & $115 / 118(97.5 \%)$ \\
\hline$[3,2]$ & $4 / 4(100 \%)$ & {$[3,1],[3,1],[3,2]$} & $378 / 378(100 \%)$ \\
\hline$[3,0],[3,0]$ & $5 / 5(100 \%)$ & {$[3,1],[3,2],[3,2]$} & $378 / 378(100 \%)$ \\
\hline$[3,0],[3,1]$ & $18 / 20(90 \%)$ & {$[3,2],[3,2],[3,2]$} & $118 / 118(100 \%)$ \\
\hline$[3,0],[3,2]$ & $20 / 20(100 \%)$ & {$[4,0]$} & $2 / 7(28.6 \%)$ \\
\hline$[3,1],[3,1]$ & $27 / 28(96.4 \%)$ & {$[4,1]$} & $12 / 16(75 \%)$ \\
\hline$[3,1],[3,2]$ & $50 / 50(100 \%)$ & {$[4,2]$} & $25 / 26(96.2 \%)$ \\
\hline$[3,2],[3,2]$ & $28 / 28(100 \%)$ & {$[4,3]$} & $16 / 16(100 \%)$ \\
\hline$[3,1],[4,0]$ & $59 / 71(83.1 \%)$ & {$[5,1]$} & $15 / 89(16.9 \%)$ \\
\hline$[3,1],[4,1]$ & $229 / 240(95.4 \%)$ & {$[5,2]$} & $136 / 172(79.1 \%)$ \\
\hline$[3,1],[4,2]$ & $355 / 364(97.5 \%)$ & {$[5,3]$} & $168 / 172(97.7 \%)$ \\
\hline$[3,0],[4,1]$ & $84 / 88(95.5 \%)$ & {$[5,4]$} & $89 / 89(100 \%)$ \\
\hline$[3,0],[4,2]$ & $133 / 136(97.8 \%)$ & & \\
\hline$[4,0],[5,1]$ & $($ partial results available) & & \\
\hline
\end{tabular}

Table 4: Success rate of schemes for various sets of barred patterns

This scheme gives the sequence $1,2,6,23,104,532,3004,18426,121393,851810$, $6325151,49448313,405298482,3470885747,30965656442$ for $S_{n}(\{25143\})$ with $1 \leqslant n \leqslant$ 15. 15

Next, the equivalence class represented by the pattern $25 \overline{1} 34$ has the scheme in Figure

This differs from the scheme for $S_{n}(\{25 \overline{1} 43\})$ only by the gap vector associated to 132 , and yields the same sequence.

The equivalence class with representative $43 \overline{5} 21$ has the scheme in Figure 16.

This is also symmetric to the previous schemes and yields the same sequence.

Finally, the equivalence class with representative $43 \overline{5} 12$ has the scheme in Figure 17.

Again, since the only difference from the scheme for $S_{n}(\{43 \overline{5} 21\})$ is the gap vector associated with prefix 321 , so we get the same sequence yet again.

Now, we consider schemes for the 4 patterns of length 5 with two bars that yield new sequences.

The pattern $5 \overline{12} 43$ has the scheme in Figure 18. This yields the new sequence 1, 2, $5,14,43,143,511,1950,7903,33848,152529,720466,3555715,18285538,97752779$ for $S_{n}(\{5 \overline{12} 43\}), 1 \leqslant n \leqslant 15$.

The equivalence class with representative $31 \overline{54} 2$ has the scheme in Figure 19, which 


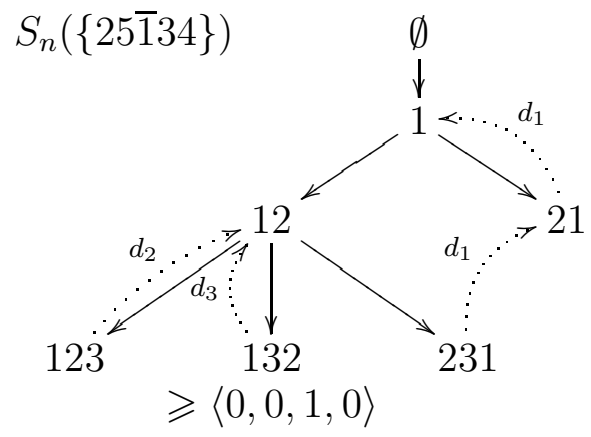

Figure 15: The scheme for $S_{n}(\{25 \overline{1} 34\})$

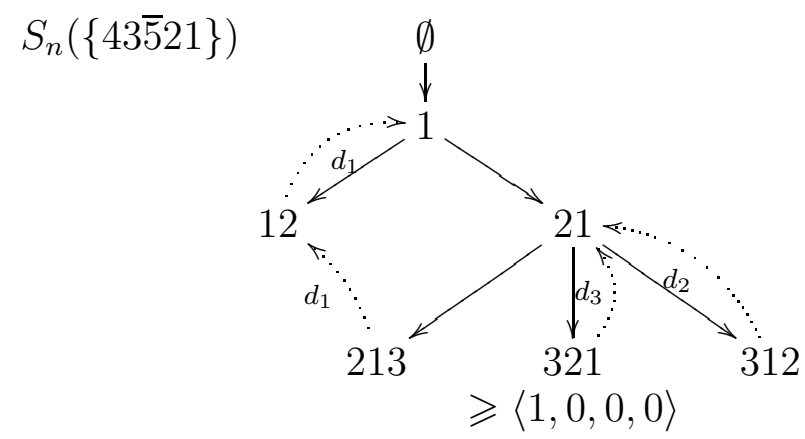

Figure 16: The scheme for $S_{n}(\{43 \overline{5} 21\})$

yields the new sequence $1,1,2,5,14,43,144,522,2030,8398,36714,168793,813112$, 4091735, 21451972, 116891160 for $S_{n}(\{31 \overline{54} 2\}), 1 \leqslant n \leqslant 15$.

The equivalence class with representative $\overline{54} 231$ has the scheme in Figure 20, which gives the new sequence 1, 2, 5, 14, 43, 146, 561, 2518, 13563, 88354, 686137, 6191526, 63330147, 720314930, 8985750097 for $S_{n}(\{\overline{54} 231\}), 1 \leqslant n \leqslant 15$.

Finally, the pattern $\overline{54} 132$ has the scheme in Figure 21, which gives the new sequence 1, 1, 2, 5, 14, 43, 147, 575, 2648, 14617, 96696, 754585, 6794015, 69116493, 781266266, 9688636317 for $S_{n}(\{\overline{54} 132\}), 1 \leqslant n \leqslant 15$.

In light of the preceding discussion, each of these schemes can be considered as a rigorously proven recurrence counting pattern-avoiding permutations, each sequence completely new to the literature. 


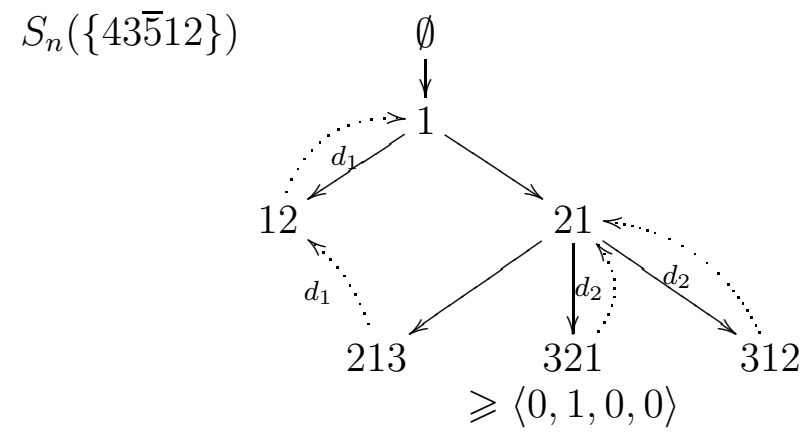

Figure 17: The scheme for $S_{n}(\{43 \overline{5} 12\})$

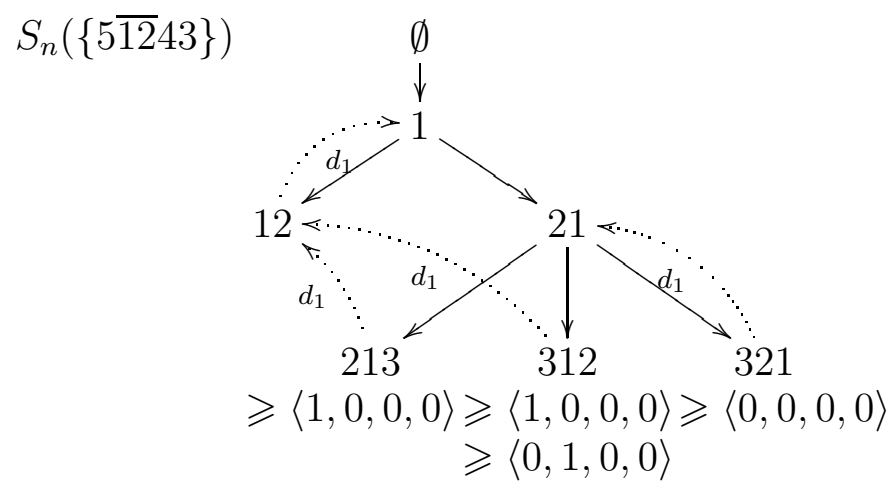

Figure 18: The scheme for $S_{n}(\{5 \overline{12} 43\})$

\section{Summary and Future Work}

Now that we have completed our discussion of permutations avoiding barred patterns, and explored the success of prefix schemes for completing this enumeration, we have discovered recurrences for several new sequences.

It still remains to determine if there are "nice" closed forms or generating functions for these sequences, and to find ways to count permutations which avoid barred patterns where enumeration schemes have not yet succeeded.

However, it is important to note that this method of enumeration schemes, already very successful for counting pattern-avoiding permutations and pattern-avoiding words in the standard sense extends nicely to enumerate permutations avoiding barred patterns as well. Moreover, this is the first such method for counting many classes of permutations avoiding barred patterns. 


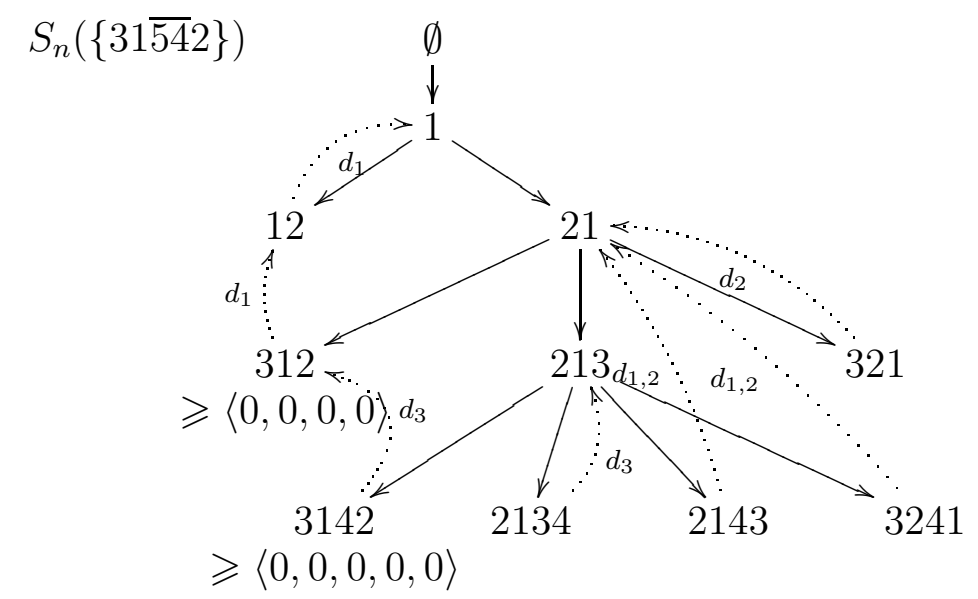

Figure 19: The scheme for $S_{n}(\{31 \overline{54} 2\})$

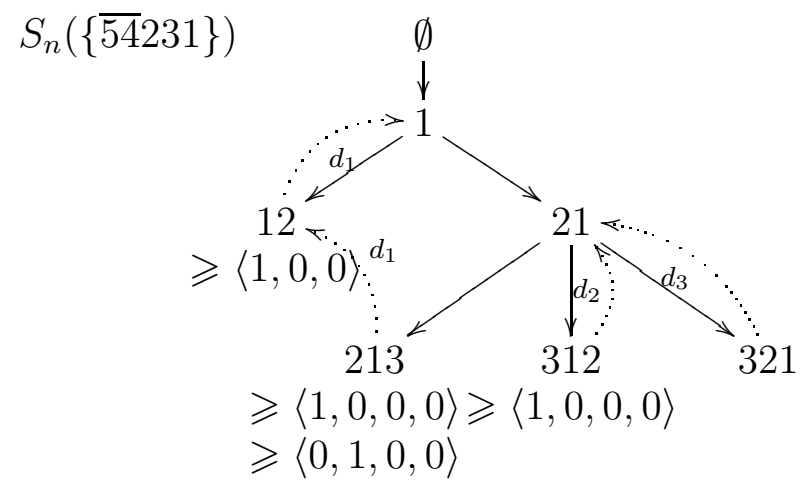

Figure 20: The scheme for $S_{n}(\{\overline{54} 231\})$

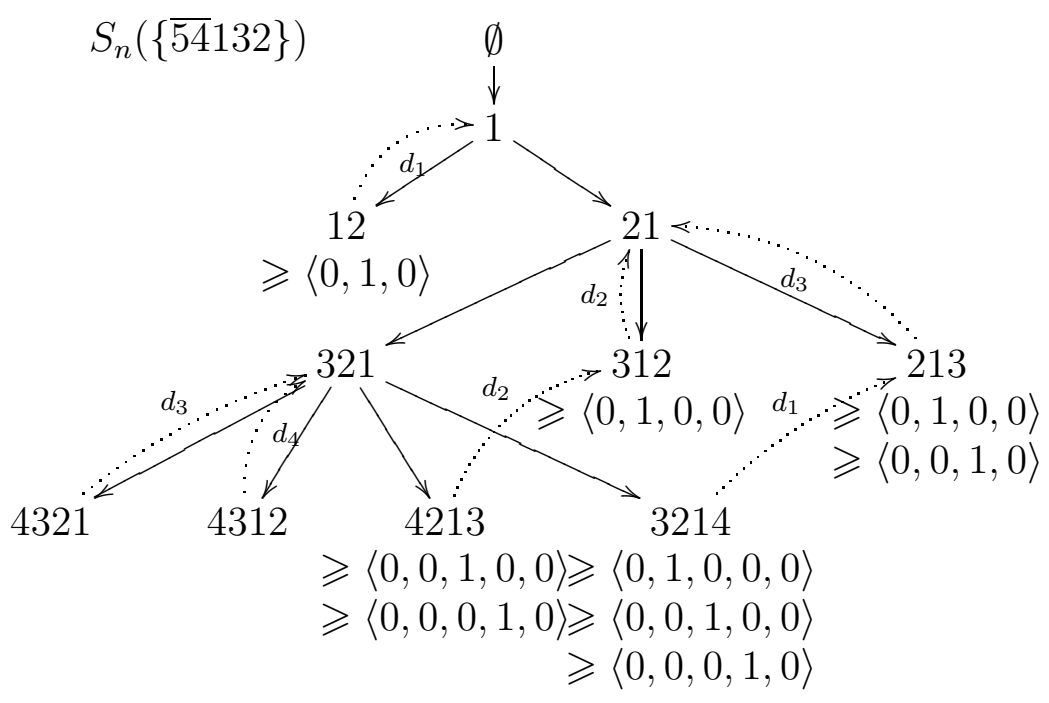

Figure 21: The scheme for $S_{n}(\{\overline{54} 132\})$ 


\section{References}

[1] M. Bona, Combinatorics of Permutations, Chapman \& Hall, 2004, Chap. 4, 129-173.

[2] S. Butler and M. Bousquet-Melou, Forest-like permutations, Ann. Combin. 11 (2007), $335-354$.

[3] D. Callan, A combinatorial interpretation for the eigensequence for composition, $J$. Integer Seq. 9 (2006), A06.1.4.

[4] D. Callan, A Wilf equivalence related to two stack sortable permutations, arXiv:math.CO/0510211v1.

[5] A. Claesson, Generalized Pattern Avoidance, European J. Combin. 22 (2001), 961971.

[6] V. Lakshmibai and B. Sandhya, Criterion for smoothness of Schubert varieties in SL $(n) /$ B, Proc. Indian Acad. Sci. Math. Sci. 100 (1990), 45-52.

[7] D. Marinov and R. Radoičić, Counting 1324-avoiding permutations, Electron. J. Combin., 9(2) (2002-2003), \#R13.

[8] R. Simion and F. W. Schmidt, Restricted permutations, European J. Combin. 6 (1985), 383-406.

[9] N. J. A. Sloane, The On-Line Encyclopedia of Integer Sequences, http://www.research.att.com/ njas/sequences/, 2008.

[10] V. Vatter, Enumeration schemes for restricted permutations, Combin. Prob. Comput. 17 (2008), 137-159.

[11] J. West, Permutations with forbidden subsequences and stack-sortable permutations, Ph.D. thesis, MIT (1990).

[12] A. Woo and A. Yong, "When is a Schubert variety Gorenstein?", Adv. Math. 207 (1) (2006), 205-220.

[13] D. Zeilberger, Enumeration schemes, and more importantly, their automatic generation, Ann. Combin. 2 (1998), 185-195.

[14] D. Zeilberger, On Vince Vatter's brilliant extension of Doron Zeilberger's enumeration schemes for Herb Wilf's classes, The Personal Journal of Ekhad and Zeilberger, 2006. http://www. math.rutgers . edu/ zeilberg/pj.html. 